\title{
Essential Oil Variation in Brazilian Varronia curassavica Jacq. in Response to Drying and Edaphoclimatic Conditions
}

\author{
Teomar Duarte da Silva ${ }^{1}$, Michele Trombin de Souza ${ }^{2}$, Mireli Trombin de Souza ${ }^{2}$, Roger Raupp Cipriano ${ }^{2}$, \\ Humberto Ribeiro Bizzo ${ }^{3} \&$ Cicero Deschamps ${ }^{2}$ \\ ${ }^{1}$ Department of Agronomy, Catarinense Federal Institute, Araquari, Brazil \\ ${ }^{2}$ Graduate Program in Agronomy-Plant Production, Federal University of Parana, Curitiba, Brazil \\ ${ }^{3}$ Embrapa Food Technology, Rio de Janeiro, Brazil \\ Correspondence: Teomar Duarte da Silva, Catarinense Federal Institute, Araquari, Santa Catarina, Highway BR \\ 280, km 27, Brazil. Tel: 55-(41)-3350-5687. E-mail: teomar.duartedasilva@gmail.com
}

Received: May 18, 2021

doi:10.5539/jas.v13n8p16
Accepted: June 25, $2021 \quad$ Online Published: July 15, 2021

URL: https://doi.org/10.5539/jas.v13n8p16

\begin{abstract}
Varronia curassavica Jacq. (Boraginaceae) is a native species of the Atlantic Forest with medical importance. This study determined the essential oil variation of 16 populations of $V$. curassavica of restinga in Santa Catarina, Brazil, in response to drying and edaphoclimatic conditions. The populations supplied essential oil with a content between 0.27 to $1.15 \%$ in the summer and from 0.33 to $1.12 \%$ in the winter. 41 chemical compounds were identified in the summer and 40 in the winter. The compounds were grouped into 4 chemical classes in each station studied. The common chemical constituents found in the essential oil of all populations and in both seasons were $\alpha$-thujene, $\alpha$-pinene, sabinene, $\alpha$-humulene, $(E)$-cariophylene, spatulenol, mircene, allo-aromadendrene, $\beta$-sesquifelandreno and $\alpha$-zingiberene. Cluster analysis using the nearest neighbor method based on Euclidean distance grouped the 16 populations into 3 groups in the summer and 8 groups in the winter. As the habitats have distinct pedological characteristics, we identified that $\mathrm{pH}$, organic matter, sum of bases and base saturation are associated with the synthesis of $(E)$-caryophyllene, $\alpha$-humulene, and allo-aromadendrene from populations.
\end{abstract}

Keywords: aromatic plant, chemical composition, phytochemical, restinga

\section{Introduction}

The Atlantic Forest is one of 25 recognized biodiversity hotspots globally; it is home to more than 19,000 species, of which 35\% are endemic (Oliveira et al., 2019; Souza et al., 2021). Despite the remarkable endemism levels that make the Atlantic Forest one of the most distinct regions in the Neotropics (Ribeiro et al., 2011; Souza et al., 2020), little is known about the aromatic plants' potential genetic resources in this biome. Among the botanical species that occur in the restinga - an ecosystem associated with the Atlantic Forest biome and established on sandy soils of marine origin-Varronia curassavica Jacq. (synonym $=$ Cordia verbenacea DC.) (Boraginaceae) is considered one of the main sources of molecules used in the treatment of inflammation, rheumatism, and ulcers (Passos et al., 2007; Roldão et al., 2008). This is due to the high diversity of secondary metabolites, specifically, the essential oils (EOs), which are synthesized and stored in the glandular trichomes present on the leaf surface (Feijó et al., 2014). In addition, the EO of this species stands out for being the first topical phytotherapeutic developed entirely in Brazil, with anti-inflammatory action (Nizio et al., 2015). With the commercial name of Acheflan ${ }^{\circledR}$ and launched by the Aché Laboratory in 2011, this phytomedicine has achieved prescription leadership in the medicinal plant segment, with over 1 million units sold, representing USD 8.1 million and accounting for $10 \%$ of industry revenue (Oliveira, 2017). Besides, V. curassavica has recognized efficacy by the Brazilian Health Regulatory Agency and appears in different official lists of the Brazilian Ministry of Health (Oliveira, 2017).

Pre-clinical studies with histamine-induced edema assays in mice have attributed to the sesquiterpene $\alpha$-humulene the role in the anti-inflammatory effect of the EO from $V$. curassavica (Fernandes et al., 2007; Passos et al., 2007). However, the $\alpha$-humulene content of this plant varies greatly (0.3-31.6\%) (Marques et al., 2019; Queiroz et al., 2020), and environmental factors must be considered to choose the best time to obtain the 
EO with the desired amount of the substance of interest. For example, the pharmaceutical industry demands that plants have a minimum content of 2.0\% $\alpha$-humulene to meet the quality standard (Magalhães, 2010).

Various environmental conditions influence the production of EOs; among them, the year-season stands out; when the season changes, plants perform physiological changes in their metabolism to amplify $\mathrm{CO}_{2}$ uptake and water and nutrient cycling, thus reflecting in the increase or decrease in the content and/or relative percentages of the compounds present in EOs (Dehsheikh et al., 2019). Some authors attribute that temperature can alter EO production via activation of thermosensitive enzymes involved in the mevalonic acid pathway, precursors of terpenes (Burbott \& Loomis, 1967; Rahimmalek \& Goli, 2013; De Almeida et al., 2016). Additionally, solar radiation can influence EO production directly or indirectly through increased plant biomass (Burbott \& Loomis, 1967). EOs production is influenced by various environmental conditions, of which the seasons of the year stand out. Season change entails physiological changes in plant metabolism to amplify $\mathrm{CO}_{2}$ uptake and water and nutrient cycling, reflecting the increase or decrease in the content and/or relative percentages of compounds present in EOs (Dehsheikh et al., 2019). Some studies have ascribed that temperature can alter EO production via activation of thermosensitive enzymes involved in the mevalonate pathway, a precursor of terpenes (Burbott \& Loomis, 1967; Rahimmalek \& Goli, 2013; De Almeida et al., 2016). Additionally, solar radiation can influence EO production directly or indirectly through increased plant biomass (Burbott \& Loomis, 1967).

Previous studies have reported that the geographic location of plants along with the soil and climate conditions serve as modulators in EO production (Rahimmalek et al., 2017; Marques et al., 2019); thus, the same species may show differential EO production depending on the environment in which it is established. Similarly, that different genotypes can result in differential essential oil production, possibly due to the behavior of floral visitors that can induce gene flow among them (Hoeltgebaum et al., 2018). Another factor is that different plant phenological stages contribute to marked differences in EOs productivity (Bouyahya et al., 2019). However, regardless of the phenological stage at which the plant is harvested, this procedure is usually performed when the plant has high water content, and drying is the most widely used process to ensure the quality and stability of EOs after harvest.

Understanding the genotype $\times$ environment interaction may provide new insights for selecting $V$. curassavica matrices with potential pharmaceutical use, which should be coordinated with sustainable use practices of the species. Although $V$. curassavica has a wide geographic distribution along the coastal zones of the Santa Catarina state in Brazil (Bayeux et al., 2002), to our knowledge, there is no information about the chemical compounds of EOs found in these populations. In addition, the diversity of EOs among plants collected in natural habitats enables determining the collecting seasons, as well as the ideal growing conditions for domestication and improvement of the plants. In this sense, our objective was to determine the chemical variation of EOs from $V$. curassavica specimens collected from 16 native restinga populations in Santa Catarina, in response to drying and edaphoclimatic conditions.

\section{Method}

The experiments were conducted under field and laboratory conditions, during summer (February 2015 and 2016) and winter (September 2015 and 2016), to determine the influence of soil and climatic conditions on the OE content and chemical composition of $V$. curassavica

\subsection{Characterization of the Collection Site}

Detailed information about the collection site of the $V$. curassavica populations used in this study is detailed in Table 1. For the soil physicochemical analyses, six soil samples were collected at a depth of $20 \mathrm{~cm}$ at each location in April 2015. The samples from each location were pooled to form a composite sample and subsequently air-dried and sieved $(1 \mathrm{~mm})$. The fraction thinner than $1 \mathrm{~mm}$ was retained for physicochemical analyses. The potential of hydrogen $(\mathrm{pH})$ was determined in a 1:1 soil-water volume ratio. Phosphorus $(\mathrm{P})$ was extracted with Mehlich solution, and Aluminum (Al) was extracted with $1 \mathrm{~mol} \mathrm{~L}^{-1}$ potassium chloride $(\mathrm{KCl})$. Organic matter (OM), base saturation (V), and the sum of bases (SB) were determined according to Silva (1999). The mean monthly values of temperature (Tp) and precipitation (Pp) in summer (February 2015 and 2016) and winter (September 2015 and 2016) were obtained from the climatological station of the Agribusiness Research and Rural Extension Company of Santa Catarina (Empresa de Pesquisa Agropecuária e Extensão Rural de Santa Catarina-EPAGRI).

\subsection{Plant Material and Isolation of the Essential Oils (EOs)}

Leaves from 16 populations of $V$. curassavica were collected along approximately $77 \mathrm{~km}$ of the Santa Catarina coast, between latitudes $26^{\circ} 22^{\prime} 22.2^{\prime \prime} \mathrm{S}$ and $26^{\circ} 51^{\prime} 52.4^{\prime \prime} \mathrm{S}$, during the summer (February 2015 and 2016) and 
winter (September 2015 and 2016). The populations were identified with codes according to the municipality where they are located (Table 1).

Table 1. Information on localities, soil and climate conditions, and size of native populations of Varronia curassavica collected in the restinga of Santa Catarina, Brazil

\begin{tabular}{|c|c|c|c|c|c|c|c|c|c|c|c|c|c|c|}
\hline Locality $^{1}$ & $\begin{array}{l}\text { Geographical } \\
\text { coordinates }\end{array}$ & $\begin{array}{l}A^{2} \\
\left(m^{2}\right)\end{array}$ & $\begin{array}{l}\mathbf{H P}^{3} \\
(\mathrm{~m})\end{array}$ & $\begin{array}{l}\text { HRF }^{4} \\
\text { (m) }\end{array}$ & $\mathrm{pH}^{5}$ & $\begin{array}{l}P^{6} \\
\left(\mathrm{mg} / \mathrm{dm}^{3}\right)\end{array}$ & $\begin{array}{l}\mathrm{OM}^{7} \\
(\%)\end{array}$ & $\mathbf{A l}^{8}$ & $\mathbf{V}^{9}$ & $\mathrm{SB}^{10}$ & $\begin{array}{l}\mathrm{TV} \mathrm{V}^{11} \\
\left({ }^{0} \mathrm{C}\right)\end{array}$ & $\begin{array}{l}\mathrm{TI}^{12} \\
\left({ }^{\circ} \mathrm{C}\right)\end{array}$ & $\begin{array}{l}P_{p V^{13}} \\
(\mathrm{~mm})^{13}\end{array}$ & $\begin{array}{l}\mathrm{PpI}^{14} \\
(\mathrm{~mm})\end{array}$ \\
\hline SF1 & $\begin{array}{l}26^{\circ} 22^{\prime} 22.2^{\prime \prime} \mathrm{S} \\
48^{\circ} 34^{\prime} 25.0^{\prime \prime} \mathrm{W}\end{array}$ & 13 & 0.47 & 0.17 & 5.8 & 6.3 & 0.2 & 0.00 & 54.14 & 1.07 & 24.7 & 17.7 & 256.3 & 107.7 \\
\hline SF2 & $\begin{array}{l}26^{\circ} 23^{\prime} 48.4^{\prime \prime} \mathrm{S} \\
48^{\circ} 35^{\prime} 16.9^{\prime \prime} \mathrm{W}\end{array}$ & 29 & 0.74 & 0.18 & 6.2 & 10.8 & 1.0 & 0.00 & 79.47 & 5.04 & 24.7 & 17.7 & 256.3 & 107.7 \\
\hline BS1 & $\begin{array}{l}26^{\circ} 27^{\prime} 20.3^{\prime \prime} \mathrm{S} \\
48^{\circ} 35^{\prime} 16.9^{\prime \prime} \mathrm{W}\end{array}$ & 15 & 0.94 & 0.27 & 5.5 & 16.8 & 0.2 & 0.00 & 62.84 & 1.35 & 24.8 & 18.2 & 256.3 & 107.7 \\
\hline BS2 & $\begin{array}{l}26^{\circ} 28^{\prime} 30.4^{\prime \prime} \mathrm{S} \\
48^{\circ} 36^{\prime} 45.6^{\prime \prime} \mathrm{W}\end{array}$ & 59 & 0.47 & 0.20 & 6.0 & 15.0 & 1.1 & 0.00 & 76.92 & 3.68 & 24.8 & 18.2 & 256.3 & 107.7 \\
\hline BS3 & $\begin{array}{l}26^{\circ} 30^{\prime} 27.2^{\prime \prime} \mathrm{S} \\
48^{\circ} 37^{\prime} 56.2^{\prime \prime} \mathrm{W}\end{array}$ & 11 & 0.51 & 0.18 & 5.7 & 9.3 & 1.0 & 0.00 & 65.70 & 2.10 & 24.8 & 18.2 & 256.3 & 107.7 \\
\hline BS4 & $\begin{array}{l}26^{\circ} 31^{\prime} 48.6^{\prime \prime} \mathrm{S} \\
48^{\circ} 38^{\prime} 36.5^{\prime \prime} \mathrm{W}\end{array}$ & 44 & 0.44 & 0.19 & 4.9 & 7.5 & 0.5 & 13.50 & 31.42 & 0.64 & 24.8 & 18.2 & 256.3 & 107.7 \\
\hline BV1 & $\begin{array}{l}26^{\circ} 35^{\prime} 26.9^{\prime \prime} \mathrm{S} \\
48^{\circ} 40^{\prime} 10.8^{\prime \prime} \mathrm{W}\end{array}$ & 9 & 0.57 & 0.19 & 4.7 & 5.9 & 0.7 & 30.47 & 34.72 & 1.60 & 22.5 & 16.2 & 202.0 & 99.3 \\
\hline BV2 & $\begin{array}{l}26^{\circ} 36^{\prime} 10.7^{\prime \prime} \mathrm{S} \\
48^{\circ} 40^{\prime} 25.2^{\prime \prime} \mathrm{W}\end{array}$ & 11 & 0.54 & 0.23 & 5.2 & 18.0 & 0.6 & 4.18 & 62.12 & 2.29 & 22.5 & 16.2 & 202.0 & 99.3 \\
\hline BV3 & $\begin{array}{l}26^{\circ} 36^{\prime} 22.6^{\prime \prime} \mathrm{S} \\
48^{\circ} 40^{\prime} 29.5^{\prime \prime} \mathrm{W}\end{array}$ & 6 & 0.54 & 0.22 & 6.2 & 11.0 & 1.1 & 0.00 & 73.96 & 3.40 & 22.5 & 16.2 & 202.0 & 99.3 \\
\hline PI1 & $\begin{array}{l}26^{\circ} 43^{\prime} 38.6^{\prime \prime} \mathrm{S} \\
48^{\circ} 40^{\prime} 51.6^{\prime \prime} \mathrm{W}\end{array}$ & 16 & 1.05 & 0.29 & 7.1 & 45.5 & 0.4 & 0.00 & 79.34 & 2.68 & 24.7 & 17.8 & 202.0 & 99.3 \\
\hline PI2 & $\begin{array}{l}26^{\circ} 44^{\prime} 04.9^{\prime \prime} \mathrm{S} \\
48^{\circ} 40^{\prime} 49.6^{\prime \prime} \mathrm{W}\end{array}$ & 8 & 1.23 & 0.26 & 5.5 & 5.0 & 1.1 & 0.00 & 69.66 & 3.68 & 24.7 & 17.8 & 202.0 & 99.3 \\
\hline PE1 & $\begin{array}{l}26^{\circ} 45^{\prime} 48.3^{\prime \prime} \mathrm{S} \\
48^{\circ} 38^{\prime} 44.8^{\prime \prime} \mathrm{W}\end{array}$ & 8 & 0.84 & 0.30 & 6.3 & 12.7 & 5.2 & 0.00 & 89.39 & 15.99 & 24.7 & 17.8 & 202.0 & 99.3 \\
\hline PE2 & $\begin{array}{l}26^{\circ} 46^{\prime} 51.8^{\prime \prime} \mathrm{S} \\
48^{\circ} 35^{\prime} 49.9^{\prime \prime} \mathrm{W}\end{array}$ & 9 & 1.23 & 0.31 & 7.8 & 9.0 & 0.1 & 0.00 & 81.97 & 2.79 & 24.7 & 17.8 & 202.0 & 99.3 \\
\hline PE3 & $\begin{array}{l}26^{\circ} 48^{\prime} 11.3^{\prime \prime} \mathrm{S} \\
48^{\circ} 35^{\prime} 49.7^{\prime \prime} \mathrm{W}\end{array}$ & 10 & 1.08 & 0.29 & 8.0 & 13.2 & 1.2 & 0.00 & 91.52 & 6.46 & 24.7 & 17.8 & 202.0 & 99.3 \\
\hline NA1 & $\begin{array}{l}26^{\circ} 51^{\prime} 28.9^{\prime \prime} \mathrm{S} \\
48^{\circ} 38^{\prime} 09.0^{\prime \prime} \mathrm{W}\end{array}$ & 18 & 0.68 & 0.24 & 5.4 & 14.3 & 0.2 & 0.00 & 51.45 & 1.48 & 24.7 & 17.8 & 202.0 & 99.3 \\
\hline NA2 & $\begin{array}{l}26^{\circ} 51^{\prime} 52.4^{\prime \prime} \mathrm{S} \\
48^{\circ} 38^{\prime} 15.0^{\prime \prime} \mathrm{W}\end{array}$ & 26 & 0.84 & 0.27 & 5.2 & 12.2 & 0.3 & 6.70 & 53.75 & 1.39 & 24.7 & 17.8 & 202.0 & 99.3 \\
\hline
\end{tabular}

Note. ${ }^{1}$ SF1, São Francisco do Sul; SF2, São Francisco do Sul; BS1, Balneário Barra do Sul; BS2, Balneário Barra do Sul; BS3, Balneário Barra do Sul; BS4, Balneário Barra do Sul; BV1, Barra Velha; BV2, Barra Velha; BV3, Barra Velha; PI1, Balneário Piçarras; PI2, Balneário Piçarras; PE1, Penha; PE2, Penha; PE3, Penha; NA1, Navegantes; NA2, Navegantes. ${ }^{2}$ Population area. ${ }^{3}$ Average plant height. ${ }^{4}$ Average height of the portion of branches with leaves. ${ }^{5}$ Potential of hydrogen. ${ }^{6}$ Phosphorus. ${ }^{7}$ Organic matter. ${ }^{8}$ Alumínio (\% saturation in cation exchange capacity). ${ }^{9}$ Percentage of base saturation. ${ }^{10}$ Base saturation. ${ }^{11}$ Average summer temperature. ${ }^{12}$ Average winter temperature. ${ }^{13}$ Average summer precipitation. ${ }^{14}$ Average winter precipitation.

On the northern coast we studied populations from São Francisco do Sul (populations SF1 and SF2), Balneário Barra do Sul (BS1, BS2, BS3 and BS4) and Barra Velha (BV1, BV2 and BV3), while on the central-northern coast we studied populations from Balneário Piçarras (PI1 and PI2), Penha (PE1, PE2 and PE3) and Navegantes (NA1 and NA2) (Table 1). Populations SF1 in the far north and NA2 in the far south are equidistant in a straight line by approximately $55 \mathrm{~km}$.

In the Atlantic Forest biome, the vegetation cover is inserted in the restinga associated ecosystem, whose ecological nature is conditioned to the vegetation complex established on sandy soils of marine origin. Each population consisted of a minimum number of 20 individuals. All individuals were selected within a maximum distance of $30 \mathrm{~cm}$ per work unit and collected on a single day. These populations were also characterized 
according to the size of their range, average plant height, and the average height of the portion of branches with leaves (Table 1).

To obtain the EOs corresponding to the fresh leaves, samples of the collected populations were immediately taken to the laboratory and subjected to hydrodistillation in a Clevenger apparatus (Vidrolabor ${ }^{\circledR}$, São Paulo, Brazil) for $3 \mathrm{~h}$. On the other hand, for the EO obtained from the dried leaves, the plant material was previously kept under a forced air circulation oven $\left(\mathrm{Fanem}^{\circledR}\right.$, São Paulo, Brazil) at $40{ }^{\circ} \mathrm{C}$ until the mass remained constant before performing the hydrodistillation process mentioned above. The EO content was determined based on dry matter basis, with three replicates per treatment. Subsequently, the EOs obtained were separated with anhydrous sodium sulfate $\left(\mathrm{Na}_{2} \mathrm{SO}_{4}\right)$ and kept refrigerated at $-4{ }^{\circ} \mathrm{C}$ in amber flasks until the chemical analyses were conducted.

\subsection{Chemical Analysis of Essential Oils (EOs)}

GC-MS was carried out in an Agilent 6890 gas chromatograph coupled to an Agilent $5973 \mathrm{~N}$ mass selective detector. The GC was fitted with an HP-5MS fused capillary column $(30 \mathrm{~m} \times 0.25 \mathrm{~mm} \times 0.25 \mu \mathrm{m}$ film thickness $)$ coated with $5 \%$ phenyl-95\% dimethylpolysiloxane stationary phase. Helium was used as the carrier gas at a flow rate of $1.0 \mathrm{~mL} / \mathrm{min}$. Temperature programming was set to $60-240{ }^{\circ} \mathrm{C}$ at the rate of $3{ }^{\circ} \mathrm{C} / \mathrm{min}$, heated to $240{ }^{\circ} \mathrm{C}$, and held at this temperature for $10 \mathrm{~min}$. The injector temperature was kept at $250^{\circ} \mathrm{C}$. Essential oil samples were diluted to a $1 \%$ solution in dichloromethane, and $1.0 \mu \mathrm{L}$ of the solution was injected with a split ratio of 1:20. The mass detector was operated in electron ionization mode $(70 \mathrm{eV})$ at a $3.15 \mathrm{scan} / \mathrm{min}$ rate and a scan range of 40-450 Da. The transfer line was maintained at $260{ }^{\circ} \mathrm{C}$, the ion source at $230{ }^{\circ} \mathrm{C}$, and the analyzer (quadrupole) at $150^{\circ} \mathrm{C}$. For the quantification, the EOs were injected in an Agilent $7890 \mathrm{~A}$ gas chromatograph fitted with FID operated at $280^{\circ} \mathrm{C}$. Hydrogen was used as the carrier gas at a flow rate of $1.5 \mathrm{~mL} / \mathrm{min}$, using the same column and conditions described above.

The quantification of each constituent was estimated by electronic integration of the FID signal with the corresponding peak area, which was determined based on the average of three injections (Tables 3 and 4). The identification of the oil's components was carried out by comparison of the mass spectra with those from commercial libraries and also by their linear retention indexes, after the injection of a homologous series of alkanes $\left(\mathrm{C}_{8}-\mathrm{C}_{26}\right)$, under the same experimental conditions, compared to literature data (Adams, 2007).

\subsection{Data Analyses}

The EOs and $\alpha$-humulene contents were submitted to the Shapiro-Wilk test to analyze the normality of the residuals and homogeneity of the variances. All data were subjected to analysis of variance (ANOVA), and the means were compared by the Scott-Knott method at 5\% error probability. For the multivariate analysis, the Euclidean distance was estimated using the 'dist' function; the HCA was performed by the Unweighted Pair Group with Arithmetic Mean (UPGMA) method through the 'hclust' function, and the PCA was performed by the 'princomp' function. All of the functions belong to the 'stats' package. All data were analyzed in the "R" statistical software version 2.15.1 (R Development Core Team 2012).

\section{Results}

EO content from the fresh and dried leaves of $V$. curassavica differed with the seasons of the year. However, SF1 and BV3 populations showed no significant variations across seasons, with EOs contents ranging from 0.9 to $1.3 \%$ and 0.3 to $0.6 \%$, respectively. In summer, EOs contents ranged from 0.3 to $1.0 \%$ in fresh leaves. After the leaves were dried, all populations maintained high EOs contents, which ranged from 0.5 to $2.7 \%$. On the other hand, in winter, EOs contents ranged from 0.3 to $1.1 \%$ in fresh leaves and from 0.2 to $1.2 \%$ in dried leaves. Under these conditions, populations SF1, SF2, PI2, PE3, and NA1 showed higher contents (Table 2). We also observed an increase in EOs content in winter compared to summer in fresh leaves of populations BV2 and PE3 (Table 2). 
Table 2. Essential oil content (\%) of native populations of Varronia curassavica collected in the restinga of Santa Catarina, Brazil

\begin{tabular}{|c|c|c|c|c|}
\hline \multirow{2}{*}{ Populations } & \multicolumn{2}{|c|}{ Summer } & \multicolumn{2}{|c|}{ Winter } \\
\hline & Fresh & Dried & Fresh & Dried \\
\hline SF1* & $0.9 \mathrm{aA}^{\#}$ & $1.3 \mathrm{cA}$ & $1.1 \mathrm{aA}$ & $1.0 \mathrm{aA}$ \\
\hline SF2 & $0.7 \mathrm{aB}$ & $1.6 \mathrm{cA}$ & $0.8 \mathrm{aB}$ & $1.1 \mathrm{aB}$ \\
\hline BS1 & $0.7 \mathrm{aB}$ & $1.5 \mathrm{cA}$ & $0.4 \mathrm{bB}$ & $0.8 \mathrm{bB}$ \\
\hline BS2 & $0.4 \mathrm{bC}$ & $1.6 \mathrm{cA}$ & $0.6 \mathrm{bB}$ & $1.0 \mathrm{aB}$ \\
\hline BS3 & $0.9 \mathrm{aB}$ & $2.0 \mathrm{bA}$ & $0.9 \mathrm{aB}$ & $0.3 \mathrm{cC}$ \\
\hline BS4 & $0.5 \mathrm{bB}$ & $1.4 \mathrm{cA}$ & $0.5 \mathrm{bB}$ & $0.7 \mathrm{bB}$ \\
\hline BV1 & $0.6 \mathrm{bB}$ & $1.6 \mathrm{cA}$ & $0.8 \mathrm{aB}$ & $0.7 \mathrm{bB}$ \\
\hline BV2 & $0.6 \mathrm{bB}$ & $1.1 \mathrm{dA}$ & $1.1 \mathrm{aA}$ & $0.7 \mathrm{bB}$ \\
\hline BV3 & $0.3 \mathrm{bA}$ & $0.5 \mathrm{eA}$ & $0.5 \mathrm{bA}$ & $0.6 \mathrm{bA}$ \\
\hline PI1 & $0.8 \mathrm{aB}$ & $2.5 \mathrm{aA}$ & $0.3 \mathrm{bB}$ & $0.5 \mathrm{bB}$ \\
\hline PI2 & $0.7 \mathrm{aC}$ & $1.7 \mathrm{bA}$ & $1.1 \mathrm{aB}$ & $1.2 \mathrm{aB}$ \\
\hline PE1 & $0.7 \mathrm{aC}$ & $2.7 \mathrm{aA}$ & $0.4 \mathrm{bC}$ & $0.9 \mathrm{aB}$ \\
\hline PE2 & $0.3 \mathrm{bB}$ & $1.8 \mathrm{bA}$ & $0.7 \mathrm{aB}$ & $0.7 \mathrm{bB}$ \\
\hline PE3 & $0.6 \mathrm{bB}$ & $1.1 \mathrm{dA}$ & $0.9 \mathrm{aA}$ & $1.3 \mathrm{aA}$ \\
\hline NA1 & $1.0 \mathrm{aB}$ & $2.0 \mathrm{bA}$ & $1.0 \mathrm{aB}$ & $1.0 \mathrm{aB}$ \\
\hline NA2 & $0.6 \mathrm{bB}$ & $1.1 \mathrm{dA}$ & $0.8 \mathrm{aB}$ & $0.2 \mathrm{cC}$ \\
\hline
\end{tabular}

Note. ${ }^{\#}$ Means followed by the same lower-case letter in the column and capital letter in the row do not differ statistically from each other by Scott Knott Test, at 5\% probability level. * Populations: SF1, São Francisco do Sul; SF2, São Francisco do Sul; BS1, Balneário Barra do Sul; BS2, Balneário Barra do Sul; BS3, Balneário Barra do Sul; BS4, Balneário Barra do Sul; BV1, Barra Velha; BV2, Barra Velha; BV3, Barra Velha; PI1, Balneário Piçarras; PI2, Balneário Piçarras; PE1, Penha; PE2, Penha; PE3, Penha; NA1, Navegantes; NA2, Navegantes.

According to the EOs chemical profiles, 22 compounds were detected in summer and 18 in winter (Tables 3 and $4)$. We found that the chemical compounds with the highest relative proportion in summer were $\alpha$-thujene $(0.7-35.3 \%), \quad \alpha$-pinene $(4.1-45.6 \%), \quad(E)$-caryophyllene $(1.5-25.4 \%), \quad$ allo-aromadendrene $(0.5-25.0 \%)$, $\alpha$-zingiberene (0.8-44.4\%), and $\beta$-sesquiphellandrene (0.8-23.0\%) (Table 3). Other compounds, like $(E)$-nerolidol $(20.9 \%)$ and $\alpha$-muurolol $(18.2 \%)$, were sampled in high amounts only in the fresh leaves of population PE1 in summer (Table 3). Moreover, $\alpha$-thujene (2.1-38.5\%), $\alpha$-pinene (2.7-42.1\%), $(E)$-caryophyllene (2.6-29.9\%), allo-aromadendrene (0.9-45.4\%), $\alpha$-zingiberene (1.4-38.8\%), $\beta$-sesquiphellandrene (1.3-22.0\%), and spathulenol (1.0-20.6\%) were present in the samples collected in the winter (Table 4).

The common chemical compounds found in all populations, regardless of season, were $\alpha$-thujene, $\alpha$-pinene, sabinene, $\alpha$-humulene, $(E)$-caryophyllene, spathulenol, myrcene, allo-aromadendrene, $\beta$-sesquiphellandrene, and $\alpha$-zingiberene (Tables 3 and 4). Drying influenced the less frequent substances in EOs. In all populations, $\gamma$-muurolene was not recorded in fresh leaves in summer but was detected in dried samples, except in populations PI1, PE1, and NA2 (Table 3). We also found that limonene was detected $77.7 \%$ more often in dried leaves in summer (Table 3). Furthermore, $\alpha$-cubebene and $\beta$-copaen- $4 \alpha$-ol were detected only in PI 2 and BV1 populations, respectively, in dried leaves in winter (Table 4). 
Table 3. Chemical composition of the essential oil of fresh and dried leaf samples from native populations of $V$. curassavica collected in the restinga of Santa Catarina, Brazil, during 2015/2016 summer

\begin{tabular}{|c|c|c|c|c|c|c|c|c|c|c|c|c|c|c|c|c|c|}
\hline \multirow{2}{*}{$\begin{array}{l}\text { Chemical } \\
\text { compounds }\end{array}$} & \multirow{2}{*}{$\begin{array}{l}{ }^{1} \mathbf{R I}{ }^{\text {cal }} \\
{ }^{2} \mathbf{R I}{ }^{\text {lit }}\end{array}$} & \multicolumn{16}{|c|}{ Relative percentage of compounds by populations and drying (\%) } \\
\hline & & SF1 & SF2 & BS1 & BS2 & BS3 & BS4 & BV1 & BV2 & BV3 & PI1 & PI2 & PE1 & PE2 & PE3 & NA1 & NA2 \\
\hline \multirow{2}{*}{$\alpha$-thujene } & 925 & $12.4^{*}$ & 7.5 & 35.3 & 9.1 & 1.7 & 11.7 & 12.5 & 17.4 & 3.1 & 13.7 & 6.5 & 1.6 & 4.4 & 1.7 & 9.7 & 5.5 \\
\hline & 924 & $6.0^{\#}$ & 10.6 & 9.1 & 4.5 & 3.9 & 6.0 & 2.7 & 10.8 & 8.2 & 10.4 & 3.4 & 1.3 & 2.2 & 0.7 & 5.9 & 5.0 \\
\hline \multirow{2}{*}{$\alpha$-pinene } & 932 & 12.5 & 37.9 & 4.1 & 24.5 & 45.6 & 29.1 & 25.7 & 10.7 & 13.0 & 16.4 & 26.9 & 12.2 & 34.7 & 34.4 & 30.2 & 20.6 \\
\hline & 932 & 17.0 & 34.6 & 12.8 & 29.6 & 15.6 & 25.0 & 7.7 & 24.9 & 19.9 & 10.0 & 19.9 & 11.3 & 26.3 & 42.5 & 19.1 & 20.2 \\
\hline \multirow{2}{*}{ sabinene } & 969 & 1.5 & 1.4 & 5.1 & 1.4 & 1.0 & 1.9 & 1.9 & 2.4 & 1.0 & 2.2 & 1.3 & 0.6 & 1.7 & 0.8 & 2.5 & 0.9 \\
\hline & 969 & 0.7 & 1.5 & 1.7 & 1.1 & 0.7 & 1.2 & 0.4 & 2.5 & 1.2 & 1.6 & 0.7 & 0.8 & 1.0 & 0.6 & 1.3 & 1.0 \\
\hline \multirow{2}{*}{$\beta$-pinene } & 974 & - & - & - & 0.5 & 4.2 & 1.6 & 4.0 & - & 5.7 & - & 3.5 & 1.4 & 7.6 & 2.5 & 4.5 & - \\
\hline & 974 & - & - & - & - & 0.8 & 2.3 & - & - & 1.1 & - & 3.0 & 2.3 & 5.0 & 2.8 & 2.8 & 0.9 \\
\hline \multirow{2}{*}{ myrcene } & 989 & 0.5 & 0.5 & 0.9 & 0.4 & 0.9 & 0.6 & 0.7 & 0.5 & 0.7 & 0.5 & 0.7 & 0.4 & 1.5 & 0.7 & 0.9 & 1.0 \\
\hline & 988 & 0.5 & 0.5 & 0.5 & 0.4 & 0.5 & 0.6 & 0.5 & 0.5 & 0.4 & 0.4 & 0.5 & 0.5 & 0.8 & 0.6 & 0.6 & 0.6 \\
\hline \multirow{2}{*}{ limonene } & 1028 & - & - & 0.5 & - & - & - & - & - & - & - & - & - & - & - & 0.8 & - \\
\hline & 1024 & 4.0 & 2.4 & 0.2 & 1.1 & 3.6 & 0.4 & - & - & - & - & - & 0.7 & - & - & 1.4 & 1.6 \\
\hline \multirow{2}{*}{$\begin{array}{l}\text { Monoterpene } \\
\text { hydrocarbons }\end{array}$} & & 26.9 & 47.3 & 45.9 & 35.9 & 53.4 & 44.9 & 44.8 & 31.0 & 23.5 & 32.8 & 38.9 & 16.2 & 49.9 & 40.1 & 48.6 & 28.0 \\
\hline & & 28.2 & 49.6 & 24.3 & 36.7 & 25.1 & 35.5 & 11.3 & 38.7 & 30.8 & 22.4 & 27.5 & 16.9 & 35.3 & 47.2 & 31.1 & 29.3 \\
\hline \multirow{2}{*}{$\alpha$-cubebene } & 1346 & - & - & - & - & - & - & - & - & - & - & 5.2 & - & - & - & 6.4 & - \\
\hline & 1345 & - & - & - & - & - & - & - & - & - & - & 5.1 & - & - & - & 4.1 & - \\
\hline \multirow{2}{*}{$\beta$-elemene } & 1387 & - & 1.9 & 3.0 & 1.5 & 1.6 & 2.3 & 0.5 & - & - & - & 1.0 & - & 3.2 & 1.5 & 1.0 & - \\
\hline & 1389 & & 3.0 & 1.1 & 2.6 & 0.4 & 1.7 & - & - & 2.3 & 0.4 & - & - & 0.3 & 0.7 & 0.7 & - \\
\hline \multirow{2}{*}{$(E)$-caryophyllene } & 1416 & 4.4 & 5.3 & 4.8 & 9.1 & 6.5 & 6.1 & 3.5 & 1.5 & 3.0 & 7.6 & 7.9 & 8.5 & 8.4 & 8.5 & 5.7 & 6.1 \\
\hline & 1419 & 9.8 & 8.4 & 21.6 & 19.9 & 6.7 & 13.2 & 2.9 & 8.3 & 15.3 & 20.9 & 19.9 & 4.1 & 25.4 & 18.3 & 7.6 & 10.7 \\
\hline & 1433 & 1.7 & 0.5 & - & 0.6 & - & 0.6 & 0.7 & 1.4 & 2.0 & 1.6 & - & - & - & - & - & 1.8 \\
\hline$\alpha$-trans-bergamotene & 1432 & 1.1 & 0.9 & 0.2 & 0.7 & 1.4 & 0.7 & 1.8 & 0.7 & 0.4 & 1.1 & 0.4 & 1.7 & - & - & 0.5 & 1.2 \\
\hline & 1450 & 1.0 & 1.2 & 1.2 & 1.8 & 1.7 & 2.0 & 1.0 & 0.3 & 0.6 & 0.9 & 2.1 & 1.8 & 1.1 & 3.2 & 1.0 & 2.0 \\
\hline & 1452 & 2.6 & 2.0 & 6.8 & 4.0 & 1.8 & 4.0 & 2.7 & 2.1 & 4.3 & 5.2 & 4.4 & 3.1 & 2.8 & 6.3 & 1.7 & 3.2 \\
\hline & 1458 & 1.3 & 14.0 & 22.7 & 15.5 & 21.1 & 10.8 & 17.0 & 11.0 & 0.6 & 0.7 & 10.8 & 15.8 & 19.2 & 12.4 & 10.8 & 1.0 \\
\hline all & 1458 & 1.3 & 20.5 & 25.0 & 14.4 & 2.6 & 7.2 & 0.5 & 19.4 & 17.0 & 1.8 & 10.2 & 0.5 & 18.1 & 15.4 & 4.6 & 1.2 \\
\hline & 1474 & - & - & - & - & - & - & - & - & - & - & - & - & - & - & - & - \\
\hline$\gamma$-muurolene & 1478 & 0.9 & 1.1 & 0.2 & 1.4 & 0.4 & 0.6 & 0.4 & 2.5 & 1.4 & - & 1.6 & - & 0.2 & 0.8 & 0.5 & - \\
\hline & 1481 & 7.2 & 2.0 & - & 2.9 & - & 3.0 & 2.6 & 5.3 & 6.0 & 4.4 & 1.1 & - & 0.5 & - & - & - \\
\hline nene & 1479 & 4.8 & 0.6 & - & 0.9 & 4.9 & 2.7 & 5.6 & 1.1 & 1.6 & 3.5 & 0.9 & 6.5 & 0.5 & 0.5 & 1.9 & 5.9 \\
\hline germ & 1489 & - & 1.0 & 1.2 & 1.0 & 1.7 & 0.8 & 0.8 & 1.0 & 0.8 & 1.7 & 2.2 & 2.8 & 1.3 & 1.0 & 1.3 & 9.4 \\
\hline germ & 1484 & 1.6 & - & 0.3 & 1.9 & - & 1.0 & - & 5.1 & 3.1 & - & 2.3 & - & - & 1.6 & - & - \\
\hline & 1494 & 24.7 & 10.1 & 3.8 & 6.3 & 3.5 & 8.0 & 10.9 & 22.3 & 35.6 & 28.2 & 5.5 & 2.0 & 5.0 & 2.7 & 2.2 & 23.3 \\
\hline & 1493 & 20.0 & 3.2 & 17.5 & 2.9 & 32.0 & 13.6 & 44.4 & 5.5 & 8.5 & 24.1 & 6.8 & 38.3 & 3.6 & 0.8 & 9.5 & 24.2 \\
\hline & 1520 & 12.5 & 4.9 & 1.1 & 4.3 & 0.8 & 4.7 & 5.5 & 10.8 & 15.8 & 12.4 & 3.3 & 2.1 & 2.1 & 2.1 & 2.0 & 13.3 \\
\hline ellandrene & 1521 & 12.3 & 8.3 & 1.1 & 2.4 & 17.9 & 7.8 & 23.0 & 13.8 & 5.3 & 13.0 & 3.8 & 20.8 & 11.1 & 1.2 & 7.1 & 15.5 \\
\hline Sesquiterpene & & 52.8 & 40.9 & 37.8 & 43.0 & 36.9 & 38.3 & 42.5 & 53.6 & 64.4 & 57.5 & 38.1 & 33.0 & 40.8 & 31.4 & 30.4 & 56.9 \\
\hline hydrocarbons & & 54.4 & 48.0 & 73.8 & 51.1 & 68.1 & 52.5 & 81.3 & 58.5 & 59.2 & 70.0 & 55.4 & 75.0 & 62.0 & 45.6 & 38.2 & 61.9 \\
\hline & 1562 & 0.5 & - & - & - & - & - & - & - & 0.5 & 0.4 & - & 20.9 & - & - & - & - \\
\hline ol & 1561 & - & - & - & - & - & - & - & - & 1.6 & - & - & - & - & - & - & - \\
\hline & 1576 & 0.5 & 2.0 & 2.7 & 2.6 & 2.4 & 1.6 & 3.4 & 1.9 & 2.6 & 0.5 & 1.7 & 0.4 & 2.2 & 5.2 & 1.2 & 1.4 \\
\hline spathulenol & 1575 & 1.0 & 1.0 & 0.2 & 1.1 & 1.0 & 0.6 & 1.1 & 1.0 & 1.0 & 0.5 & 0.5 & 1.3 & 1.6 & 0.9 & 0.9 & 0.9 \\
\hline th & 1580 & 0.8 & 1.2 & - & - & - & 1.2 & - & - & 0.4 & - & - & - & - & - & - & 1.5 \\
\hline ar-turmerol & 1582 & 0.9 & - & 1.2 & 1.1 & 0.5 & 1.1 & 2.0 & 1.0 & 1.0 & 1.2 & 0.8 & - & 0.3 & 1.3 & 0.9 & 0.6 \\
\hline & 1600 & - & 0.9 & 1.5 & 0.9 & 1.1 & 0.7 & 1.3 & 0.8 & - & - & 1.0 & - & 1.1 & 1.4 & 1.0 & - \\
\hline ledol & 1602 & - & - & - & 0.7 & - & - & - & - & 0.9 & - & 0.8 & - & 0.2 & 0.7 & 1.3 & - \\
\hline & 1644 & - & - & - & - & - & 0.4 & - & 0.5 & 0.4 & - & 0.4 & 18.2 & 0.5 & 0.4 & - & - \\
\hline$\alpha$-muurolol & 1644 & 0.8 & - & 0.5 & 0.9 & 0.9 & 0.8 & 0.4 & - & 0.6 & - & 0.4 & - & 0.3 & 0.6 & 1.0 & 0.8 \\
\hline Oxygenated & & 1.8 & 4.1 & 4.2 & 3.5 & 3.5 & 3.9 & 4.7 & 3.2 & 3.9 & 0.9 & 3.1 & 39.5 & 3.8 & 7.0 & 2.2 & 2.9 \\
\hline sesquiterpenes & & 2.7 & 1.0 & 1.9 & 38.0 & 2.4 & 2.5 & 3.5 & 2.0 & 5.1 & 1.7 & 2.5 & 1.3 & 2.4 & 3.5 & 4.1 & 2.3 \\
\hline & & 81.5 & 92.3 & 87.9 & 82.4 & 93.8 & 87.1 & 92.0 & 87.8 & 91.8 & 91.2 & 80.1 & 88.7 & 94.5 & 78.5 & 81.2 & 87.8 \\
\hline identified (\%) & & 85.3 & 98.6 & 100.0 & 91.6 & 95.6 & 90.5 & 96.1 & 99.2 & 95.1 & 94.1 & 85.4 & 93.2 & 99.7 & 96.3 & 73.4 & 93.5 \\
\hline
\end{tabular}

Note. ${ }^{1} \mathrm{RI}^{\mathrm{cal}}=$ Experimental Retention Index, ${ }^{2} \mathrm{RI}{ }^{\mathrm{lit}}=$ Literature Retention Index, Populations: SF1, São Francisco do Sul; SF2, São Francisco do Sul; BS1, Balneário Barra do Sul; BS2, Balneário Barra do Sul; BS3, Balneário Barra do Sul; BS4, Balneário Barra do Sul; BV1, Barra Velha; BV2, Barra Velha; BV3, Barra Velha; PI1, Balneário Piçarras; PI2, Balneário Piçarras; PE1, Penha; PE2, Penha; PE3, Penha; NA1, Navegantes; NA2; Navegantes; * Summer. " Winter. - content lower than $0.1 \%$. 
Table 4. Chemical composition of the essential oil of fresh and dried leaf samples from native populations of $V$. curassavica collected in the restinga of Santa Catarina, Brazil, during 2015/2016 winter

\begin{tabular}{|c|c|c|c|c|c|c|c|c|c|c|c|c|c|c|c|c|c|}
\hline \multirow{2}{*}{$\begin{array}{l}\text { Chemical } \\
\text { compounds }\end{array}$} & \multirow{2}{*}{$\begin{array}{l}{ }^{1} \mathbf{R I}^{\mathrm{cal}} \\
{ }^{2} \mathbf{R I}^{\mathrm{lit}}\end{array}$} & \multicolumn{16}{|c|}{ Relative percentage of compounds by populations and drying (\%) } \\
\hline & & SF1 & SF2 & BS1 & BS2 & BS3 & BS4 & BV1 & BV2 & BV3 & PI1 & PI2 & PE1 & PE2 & PE3 & NA1 & NA2 \\
\hline \multirow{2}{*}{$\alpha$-thujene } & 925 & $9.6^{*}$ & 21.1 & 9.3 & 5.4 & 10.3 & 15.2 & 10.2 & 38.5 & 5.8 & 8.4 & 7.2 & 12.9 & 6.9 & 5.9 & 13.2 & 19.3 \\
\hline & 924 & $5.8^{\#}$ & 20.5 & 8.2 & 3.6 & 3.1 & 5.3 & 3.6 & 36.0 & 3.8 & 9.2 & 2.1 & 9.2 & 3.2 & 2.4 & 5.2 & 10.9 \\
\hline \multirow{2}{*}{$\alpha$-pinene } & 932 & 10.1 & 42.1 & 28.5 & 7.6 & 7.8 & 9.2 & 12.3 & 9.7 & 8.9 & 7.3 & 5.3 & 18.2 & 7.8 & 4.6 & 10.2 & 11.4 \\
\hline & 932 & 14.6 & 31.0 & 16.8 & 5.1 & 15.6 & 15.3 & 8.9 & 7.6 & 10.2 & 9.4 & 7.8 & 13.3 & 9.2 & 2.7 & 8.3 & 9.7 \\
\hline \multirow{2}{*}{ sabinene } & 969 & 2.6 & 3.4 & 3.5 & 2.3 & 3.2 & 3.2 & 1.1 & 1.3 & 2.4 & 5.8 & 3.3 & 2.4 & 2.9 & 3.1 & 4.6 & 3.1 \\
\hline & 969 & 5.2 & 2.8 & 8.6 & 4.3 & 1.4 & 2.7 & 2.0 & 2.9 & 1.8 & 8.4 & 3.5 & 2.0 & 4.5 & 2.5 & 6.5 & 2.5 \\
\hline \multirow{2}{*}{$\beta$-pinene } & 974 & 2.2 & - & - & - & - & 4.1 & 3.6 & - & 3.4 & 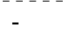 & 5.6 & 3.2 & 9.8 & - & 2.5 & - \\
\hline & 974 & 0.8 & - & - & - & - & 2.9 & - & - & 1.6 & - & 2.7 & 1.1 & 4.7 & 2.4 & 3.3 & - \\
\hline \multirow{2}{*}{ myrcene } & 989 & 4.3 & 1.0 & 3.8 & 2.6 & 2.0 & 1.0 & 1.6 & 1.2 & 1.7 & 4.8 & 2.1 & 1.0 & 9.5 & 2.7 & 1.2 & 4.2 \\
\hline & 988 & 1.5 & 1.2 & 6.7 & 4.5 & 3.1 & 1.6 & 2.5 & 1.0 & 2.4 & 6.3 & 1.0 & 0.7 & 8.4 & 3.6 & 6.6 & 2.2 \\
\hline \multirow{2}{*}{$\begin{array}{l}\text { Monoterpene } \\
\text { hydrocarbons }\end{array}$} & & & 28.8 & 67.6 & 45.1 & 17.9 & 23.3 & 32.7 & 28.8 & 50.7 & 22.2 & 26.3 & 23.5 & 37.7 & 36.9 & 16.3 & 31.7 \\
\hline & & & 27.9 & 55.5 & 40.3 & 17.5 & 23.2 & 27.8 & 17.0 & 47.5 & 19.8 & 33.3 & 17.1 & 26.3 & 30.0 & 13.6 & 29.9 \\
\hline \multirow{2}{*}{$\alpha$-cubebene } & 1346 & - & - & - & - & - & - & - & - & - & - & - & - & - & - & - & - \\
\hline & 1345 & - & - & - & - & - & - & - & - & - & - & 8.4 & - & - & - & - & - \\
\hline \multirow{2}{*}{$\beta$-elemene } & 1387 & 0.3 & 2.4 & 6.4 & 3.5 & - & 4.3 & 3.5 & - & - & - & 2.2 & - & 3.5 & 2.5 & 2.1 & - \\
\hline & 1389 & - & 1.9 & 6.8 & 2.1 & - & 5.2 & - & - & 3.8 & 4.4 & - & - & 1.2 & 3.7 & 5.9 & - \\
\hline \multirow{2}{*}{$(E)$-caryophyllene } & 1416 & 6.4 & 4.5 & 4.8 & 20.8 & 5.1 & 5.4 & 8.5 & 4.2 & 28.5 & 8.6 & 27.9 & 9.4 & 9.2 & 20.5 & 5.5 & 6.5 \\
\hline & 1419 & 4.8 & 6.0 & 9.6 & 29.9 & 8.2 & 2.6 & 6.9 & 7.6 & 25.5 & 9.9 & 24.2 & 8.1 & 7.5 & 18.3 & 9.1 & 5.8 \\
\hline \multirow{2}{*}{$\alpha$-humulene } & 1450 & 1.0 & 0.8 & 1.3 & 1.2 & 1.2 & 2.1 & 1.0 & 0.5 & 1.1 & 0.3 & 1.1 & 1.6 & 1.3 & 2.0 & 2.8 & 1.2 \\
\hline & 1452 & 1.4 & 1.0 & 2.5 & 1.1 & 1.5 & 2.2 & 1.2 & 5.0 & 2.7 & 3.2 & 2.1 & 1.8 & 1.2 & 3.1 & 2.4 & 1.0 \\
\hline \multirow{2}{*}{ allo-aromadendrene } & 1458 & 2.6 & 6.9 & 7.3 & 25.1 & 6.7 & 5.8 & 7.0 & 8.6 & 22.4 & 7.1 & 14.8 & 1.9 & 5.2 & 32.4 & 9.2 & 2.5 \\
\hline & 1458 & 0.9 & 12.4 & 9.1 & 24.3 & 5.4 & 8.9 & 3.1 & 7.2 & 17.5 & 6.8 & 33.5 & 3.4 & 4.1 & 45.4 & 5.6 & 4.9 \\
\hline \multirow{2}{*}{ arcurcumene } & 1481 & 8.9 & 2.1 & - & 4.5 & - & 2.4 & 2.1 & 7.4 & 6.3 & 7.4 & 1.1 & - & 6.2 & - & - & - \\
\hline & 1479 & 5.4 & 3.2 & 3.2 & 3.8 & 5.3 & 3.3 & 2.3 & 0.8 & 2.4 & 8.9 & 0.9 & 7.9 & 8.7 & 2.1 & 5.2 & 7.6 \\
\hline \multirow{2}{*}{$\alpha$-zingiberene } & 1494 & 23.5 & 5.6 & 4.6 & 5.2 & 25.5 & 17.6 & 13.7 & 6.5 & 7.4 & 8.4 & 3.1 & 16.2 & 4.3 & 2.5 & 13.5 & 23.2 \\
\hline & 1493 & 32.4 & 1.4 & 5.1 & 3.6 & 20.4 & 12.1 & 38.8 & 7.6 & 8.9 & 7.6 & 2.5 & 25.7 & 5.4 & 1.6 & 10.5 & 20.2 \\
\hline \multirow{2}{*}{$\beta$-sesquiphellandrene } & 1520 & 16.6 & 4.7 & 3.4 & 3.1 & 16.3 & 14.2 & 15.5 & 6.7 & 2.6 & 9.2 & 1.3 & 16.4 & 2.8 & 2.5 & 16.8 & 14.3 \\
\hline & 1521 & 18.9 & 9.2 & 5.8 & 2.9 & 14.2 & 11.4 & 22.0 & 2.3 & 5.3 & 9.8 & 3.2 & 20.3 & 9.4 & 2.2 & 12.9 & 10.2 \\
\hline Sesquiterpene & & 59.3 & 27.0 & 27.8 & 63.4 & 54.8 & 51.8 & 51.3 & 33.9 & 68.3 & 41.0 & 51.5 & 45.5 & 32.5 & 62.4 & 49.9 & 47.7 \\
\hline hydrocarbons & & 63.8 & 35.1 & 42.1 & 67.7 & 55.0 & 45.7 & 74.3 & 30.5 & 66.1 & 50.6 & 74.8 & 67.2 & 37.5 & 76.4 & 51.6 & 49.7 \\
\hline & 1576 & 6.4 & 4.0 & 2.7 & 1.0 & 3.6 & 3.8 & 5.2 & 3.2 & 3.2 & 12.5 & 3.2 & 4.0 & 15.3 & 1.6 & 3.4 & 2.1 \\
\hline & 1575 & 5.6 & 2.1 & 4.6 & 1.0 & 2.2 & 7.6 & 3.2 & 1.9 & 2.0 & 10.6 & 2.4 & 1.2 & 20.6 & 2.3 & 4.2 & 1.6 \\
\hline & 1580 & 1.0 & - & 3.8 & - & - & 2.1 & - & - & 1.4 & - & - & - & - & - & - & 1.0 \\
\hline & 1582 & - & - & 5.6 & 2.4 & 1.0 & 1.9 & - & 1.3 & 1.1 & 3.4 & - & - & 0.3 & 1.1 & 2.7 & 4.9 \\
\hline & 1600 & - & - & 6.3 & 2.2 & 3.4 & 1.2 & 2.9 & 1.9 & - & - & 1.0 & - & 1.4 & 1.4 & 2.1 & - \\
\hline le & 1602 & - & 2.2 & 5.8 & 3.7 & - & - & - & 0.2 & 1.5 & - & - & - & 2.1 & 3.7 & 3.2 & - \\
\hline & 1575 & - & - & 3.4 & 2.6 & - & 4.3 & - & 3.2 & 2.6 & 6.5 & 2.3 & 4.2 & - & 2.5 & 4.2 & 3.2 \\
\hline hyllene oxide & 1582 & - & 1.9 & - & 5.0 & - & 6.5 & - & - & - & - & - & - & - & - & - & - \\
\hline & 1592 & - & - & - & - & - & - & - & - & - & -- & - & - & - & - & - & $\begin{array}{l}-- \\
-\end{array}$ \\
\hline copaen-4 $\alpha$-ol & 1590 & - & - & - & - & - & - & 5.1 & - & - & - & - & - & - & - & - & - \\
\hline Oxygenated & & 7.4 & 4.0 & 16.2 & 5.8 & 7.0 & 11.4 & 8.1 & 8.3 & 7.2 & 19.0 & 6.5 & 8.2 & 16.7 & 5.5 & 9.7 & 6.3 \\
\hline sesquiterpenes & & 6.6 & 6.2 & 16.0 & 12.1 & 3.2 & 16.0 & 8.3 & 3.4 & 4.6 & 14.0 & 2.4 & 1.2 & 23.0 & 7.1 & 10.1 & 6.5 \\
\hline & & 95.5 & 98.6 & 89.1 & 87.1 & 85.1 & 95.9 & 88.2 & 92.9 & 97.7 & 86.3 & 81.5 & 91.4 & 86.1 & 84.2 & 91.3 & 92.0 \\
\hline ed $(\%)$ & & 98.3 & 96.8 & 98.4 & 97.3 & 81.4 & 89.5 & 99.6 & 81.4 & 90.5 & 95.9 & 94.3 & 94.7 & 90.5 & 97.1 & 91.6 & 81.5 \\
\hline
\end{tabular}

Note ${ }^{1} \mathrm{RI}^{\mathrm{cal}}=$ Experimental Retention Index, ${ }^{2} \mathrm{RI}^{\mathrm{lit}}=$ Literature Retention Index, Populations: SF1, São Francisco do Sul; SF2, São Francisco do Sul; BS1, Balneário Barra do Sul; BS2, Balneário Barra do Sul; BS3, Balneário Barra do Sul; BS4, Balneário Barra do Sul; BV1, Barra Velha; BV2, Barra Velha; BV3, Barra Velha; PI1, Balneário Piçarras; PI2, Balneário Piçarras; PE1, Penha; PE2, Penha; PE3, Penha; NA1, Navegantes; NA2; Navegantes; * Summer. ${ }^{\#}$ Winter. - content lower than $0.1 \%$.

We also found that $\alpha$-humulene was detected in $100 \%$ of the samples (Tables 3 and 4), with contents ranging from 0.3 to $6.8 \%$ (Table 5). In the comparison between populations, PE3 obtained the highest EOs contents (2.0 to $6.8 \%$ ), except in the fresh leaves of the BS4 population collected in winter $(2.1 \%)$, where these populations were similar (Table 5). After the leaves were dried, all populations collected in summer showed high contents of $\alpha$-humulene, varying from 1.7 to $6.8 \%$ (Table 5 ). 
Table 5. Content of $\alpha$-humulene (\%) in the essential oil of native populations of Varronia curassavica collected in the restingas of Santa Catarina, Brazil

\begin{tabular}{|c|c|c|c|c|}
\hline \multirow{2}{*}{ Populations } & \multicolumn{2}{|c|}{ Summer } & \multicolumn{2}{|c|}{ Winter } \\
\hline & Fresh & Dried & Fresh & Dried \\
\hline SF1* & $1.0 \mathrm{gC}^{\#}$ & $2.6 \mathrm{hA}$ & $1.0 \mathrm{eC}$ & $1.4 \mathrm{gB}$ \\
\hline $\mathrm{SF} 2$ & $1.2 \mathrm{fB}$ & $2.0 \mathrm{jA}$ & $0.8 \mathrm{fD}$ & $1.0 \mathrm{iC}$ \\
\hline BS1 & $1.2 \mathrm{fD}$ & $6.3 \mathrm{bA}$ & $1.3 \mathrm{cC}$ & $2.5 \mathrm{cB}$ \\
\hline $\mathrm{BS} 2$ & $1.8 \mathrm{~dB}$ & $4.0 \mathrm{eA}$ & $1.2 \mathrm{dC}$ & $1.1 \mathrm{hD}$ \\
\hline BS3 & $1.7 \mathrm{eB}$ & $1.81 \mathrm{~A}$ & $1.2 \mathrm{dD}$ & $1.5 \mathrm{gC}$ \\
\hline BS4 & $2.0 \mathrm{cD}$ & $4.0 \mathrm{eA}$ & $2.1 \mathrm{aC}$ & $2.2 \mathrm{~dB}$ \\
\hline BV1 & $1.0 \mathrm{gC}$ & $2.7 \mathrm{gA}$ & $1.0 \mathrm{eC}$ & $1.2 \mathrm{hB}$ \\
\hline BV2 & $0.3 \mathrm{jD}$ & $2.1 \mathrm{iA}$ & $0.5 \mathrm{gC}$ & $1.0 \mathrm{iB}$ \\
\hline BV3 & $0.6 \mathrm{iD}$ & $4.3 \mathrm{dA}$ & $1.1 \mathrm{eC}$ & $2.7 \mathrm{bB}$ \\
\hline PI1 & $0.9 \mathrm{hC}$ & $5.2 \mathrm{cA}$ & $0.3 \mathrm{hD}$ & $2.2 \mathrm{~dB}$ \\
\hline PI2 & $2.1 \mathrm{bB}$ & $4.4 \mathrm{dA}$ & $1.1 \mathrm{dD}$ & $2.1 \mathrm{eC}$ \\
\hline PE1 & $1.8 \mathrm{~dB}$ & $3.1 \mathrm{fA}$ & $1.6 \mathrm{bC}$ & $1.8 \mathrm{fB}$ \\
\hline PE2 & $1.1 \mathrm{gD}$ & $2.8 \mathrm{gA}$ & $1.3 \mathrm{cB}$ & $1.2 \mathrm{hC}$ \\
\hline PE3 & $3.2 \mathrm{aB}$ & $6.8 \mathrm{aA}$ & $2.0 \mathrm{aD}$ & $3.1 \mathrm{aC}$ \\
\hline NA1 & $1.0 \mathrm{gB}$ & $1.7 \mathrm{~mA}$ & $0.8 \mathrm{fC}$ & $0.6 \mathrm{jD}$ \\
\hline NA2 & $2.0 \mathrm{cB}$ & $3.2 \mathrm{fA}$ & $1.2 \mathrm{dC}$ & $1.0 \mathrm{iD}$ \\
\hline
\end{tabular}

Note. ${ }^{\#}$ Means followed by the same lower-case letter in the column and capital letter in the row do not differ statistically from each other by Scott Knott Test, at 5\% probability level. * Populations: SF1, São Francisco do Sul; SF2, São Francisco do Sul; BS1, Balneário Barra do Sul; BS2, Balneário Barra do Sul; BS3, Balneário Barra do Sul; BS4, Balneário Barra do Sul; BV1, Barra Velha; BV2, Barra Velha; BV3, Barra Velha; PI1, Balneário Piçarras; PI2, Balneário Piçarras; PE1, Penha; PE2, Penha; PE3, Penha; NA1, Navegantes; NA2; Navegantes.

Chemical similarities between Eos extracted from populations harvested in summer and winter were investigated by multivariate analysis of the common chemical compounds, regardless of leaf drying. HCA using the Euclidean distance of 20 units as a measure of dissimilarity showed the formation of three clusters for the summer season (Fig. 1A). Cluster 1 comprised the populations SF1, PI1, NA2, BV2, and SF2, with $\alpha$-thujene (5.0-17.4\%), $\alpha$-pinene $(10.0-37.9 \%), \quad(E)$-caryophyllene $(1.5-20.9 \%)$, allo-aromadendrene $(0.7-20.5 \%)$, $\alpha$-zingiberene (3.2-28.2\%), and $\beta$-sesquiphellandrene $(4.9-15.5 \%)$ as the main compounds. Cluster 2 encompassed the populations BV2, PE2, PE3, BS2, PI2, NA1, BS4, BV1, BS3, and P1, characterized by the main compounds $\alpha$-thujene $(0.7-17.4 \%), \quad \alpha$-pinene $\quad(7.7-45.6 \%), \quad(E)$-caryophyllene $\quad(1.5-25.4 \%)$, allo-aromadendrene (0.5-21.1\%), $\alpha$-zingiberene (2.2-44.4\%), and $\beta$-sesquiphellandrene (0.8-23.0\%). Cluster 3 was represented by the population BS1, with $\alpha$-thujene (35.3\%), $\alpha$-pinene $(12.8 \%),(E)$-caryophyllene $(21.6 \%)$, allo-aromadendrene (25.0\%), and $\alpha$-zingiberene (17.5\%) (Figure 1A).

In the winter season, we found eight clusters, using the Euclidean distance of 12 units as a dissimilarity measure (Figure 1B). Cluster 1 was formed by the populations SF1, NA2, BS3, BV1, and PE1, characterized by $\alpha$-thujene (3.1-19.3\%), $\alpha$-pinene (7.8-18.2\%), $\alpha$-zingiberene (13.7-38.8\%), and $\beta$-sesquiphellandrene (10.2-22.0\%). Cluster 2 was composed of the BS4 and NA1 populations, with $\alpha$-thujene (5.2-15.2\%), $\alpha$-pinene (8.3-15.3\%), $\alpha$-zingiberene (10.5-17.6\%), and $\beta$-sesquiphellandrene (11.4-16.8\%) among the major compounds. Cluster 3 comprised the populations BS2, BV3, PI2, and PE3, characterized by $(E)$-caryophyllene (18.3-29.9\%) and allo-aromadendrene (14.8-45.4\%). Finally, clusters 4, 5, 6, 7, and 8 were formed by one population each. In these populations, the main compounds were spathulenol (15.3-20.6\%) in PE2 (Cluster 4); spathulenol $(10.6-12.5 \%)$ in PI1 (Cluster 5); $\alpha$-pinene (16.8-28.5\%) in BS1 (Cluster 6); $\alpha$-thujene (36.0-38.5\%) in BV2 (Cluster 7); $\alpha$-thujene (20.5-21.1\%) and $\alpha$-pinene (31.0-42.1\%) in SF2 (Cluster 8) (Figure 1B). 

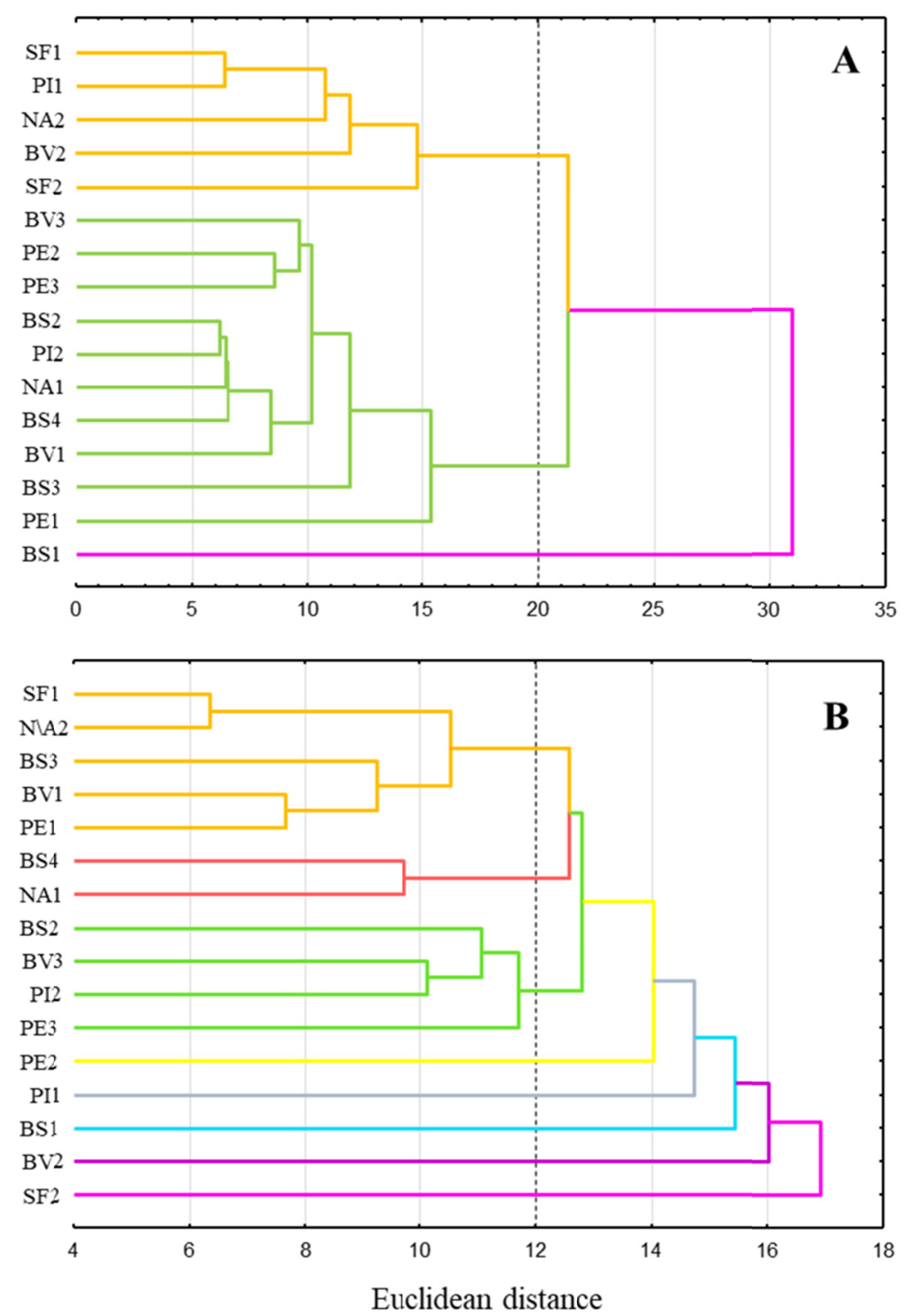

Figure 1. Hierarchical cluster analysis (HCA) of the common chemical compounds identified in the essential oils obtained from 16 native populations of Varronia curassavica collected in the restinga of Santa Catarina, Brazil, during in summer (A) and winter (B)

Note. A, summer; B, winter; Different colors in each figure represent the cluster formation. SF1, São Francisco do Sul; SF2, São Francisco do Sul; BS1, Balneário Barra do Sul; BS2, Balneário Barra do Sul; BS3, Balneário Barra do Sul; BS4, Balneário Barra do Sul; BV1, Barra Velha; BV2, Barra Velha; BV3, Barra Velha; PI1, Balneário Piçarras; PI2, Balneário Piçarras; PE1, Penha; PE2, Penha; PE3, Penha; NA1, Navegantes; NA2; Navegantes.

PCA was in line with the HCA results in most cases (Fig. 2). PCA analysis clustered the relationships between populations and the interrelationships between chemical compounds and soil and climate conditions those that showed similarities. In the summer season, the first principal component accounted for $41.5 \%$ of the total variation in the data, the compounds $\beta$-sesquiphellandrene, $\alpha$-zingiberene, $\alpha$-thujene, and $\alpha$-pinene were associated to Cluster 1 and were inversely related to the compounds $(E)$-caryophyllene, $\alpha$-humulene, and allo-aromadendrene associated to Cluster 2. According to PCA, the presence of such compounds in Cluster 2 is related to the soil and climate variables $\mathrm{Tp}, \mathrm{SB}, \mathrm{OM}, \mathrm{pH}, \mathrm{V}$, and $\mathrm{Pp}$. Cluster 1 and Cluster 3 are related to the $\mathrm{P}$ and $\mathrm{Al}$ variables (Figure $2 \mathrm{~A}$ ). The second principal component explained $23.1 \%$ of the total variation in the data, and $\alpha$-pinene, myrcene, and spathulenol were related to the soils with lower P concentration (Figure 2A). 

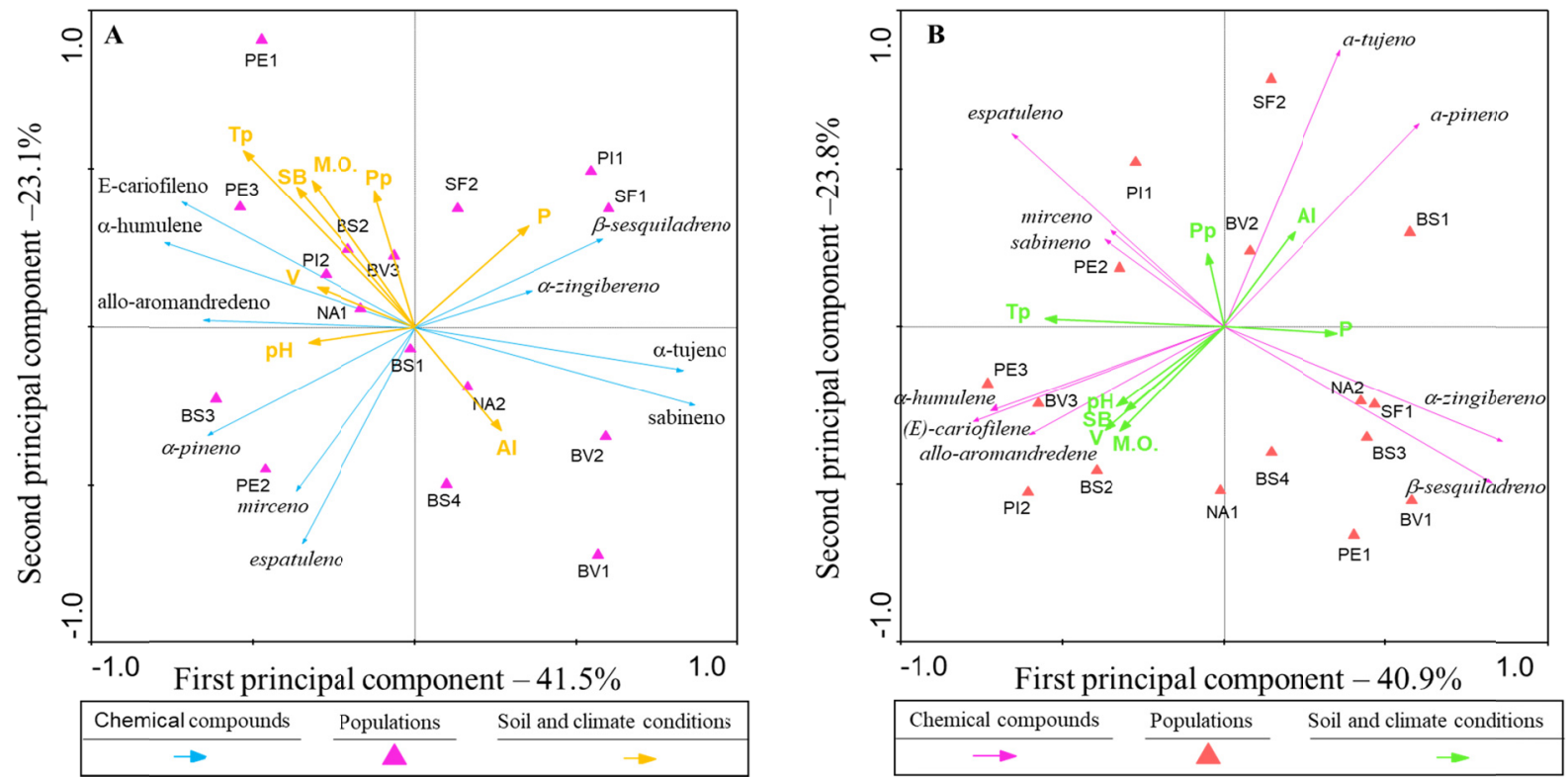

Figure 2. Principal Component Analysis (PCA) of the common chemical compounds identified in the essential oils obtained from 16 native populations of Varronia curassavica collected in the restinga of Santa Catarina, Brazil, during in summer (A) e winter (B)

Note. A, summer; B, winter; Tp, temperature; Pp, precipitation; Al, aluminum; OM, organic matter; $\mathrm{P}$, phosphorus; $\mathrm{pH}$, potential of hydrogen; SB, sum of bases; V, base saturation; SF1, São Francisco do Sul; SF2, São Francisco do Sul; BS1, Balneário Barra do Sul; BS2, Balneário Barra do Sul; BS3, Balneário Barra do Sul; BS4, Balneário Barra do Sul; BV1, Barra Velha; BV2, Barra Velha; BV3, Barra Velha; PI1, Balneário Piçarras; PI2, Balneário Piçarras; PE1, Penha; PE2, Penha; PE3, Penha; NA1, Navegantes; NA2; Navegantes.

For the winter season, $\mathrm{Tp}$ and $\mathrm{P}$ concentration were correlated with the first principal component, which explained $40.9 \%$ of the total variation in the data, with $\mathrm{Tp}$ influencing the increase in spathulenol and $\mathrm{P}$ increasing $\beta$-sesquiphellandrene and $\alpha$-zingiberene (Figure 2 B). Populations PE3, BV3, BS2, and PI2 correlate positively with soil conditioning factors such as $\mathrm{pH}, \mathrm{OM}, \mathrm{SB}$, and $\mathrm{V}$, which are positively associated with (E)-caryophyllene, $\alpha$-humulene and $\alpha$-pinene, and negatively with $\alpha$-thujene and sabinene, explaining $23.8 \%$ of the variance of the second principal component (Figure 2B).

\section{Discussion}

The EOs synthesized in the glandular trichomes of $V$. curassavica represent a mediation between plants and their surrounding environment. For the first time, our results showed that the EOs of this species collected from native populations of restinga in Santa Catarina, southern Brazil, exhibit different responses to soil and climate conditions. The SF1 and BV3 populations did not show variations in EOs content $(0.9-1.3 \%$ and $0.3-0.6 \%$, respectively). Although EO biosynthesis is determined by genetics, in the other populations studied, the variations in EO content suggest they have a geographical origin. Previous studies in other Brazilian states found that the EO content from V. curassavica populations ranged from $0.4 \%$ in São Paulo to $2.7 \%$ in Bahia, respectively (Marques et al., 2019). Queiroz et al. (2020) also showed differences in EO content (0.1-1.2\%) in a germplasm bank of $V$. curassavica cultivated in Minas Gerais. On the other hand, analyses of populations of this species collected in San Rafael-Coxcatlán, Puebla, Mexico resulted in the lowest contents ever reported in the literature $(0.1-0.3 \%)$ (Hernández et al., 2014). These comparisons suggest that some populations from Santa Catarina, such as PI1 (2.5\%) and PE1 (2.7\%), can be introduced as high EO content matrices for pharmaceutical industries. These responses in EO production benefit the selection of matrices used to determine growing conditions and improve productivity.

We observed that all populations increased EO content in the summer after the leaves were dried. This is due to the lower amount of water found in the dried samples that allows the vapor flow generated during hydrodistillation to drag the EOs stored in the plant tissues more efficiently. Evidence also indicates that the low water content in the dried plants decreases the tendency for the oil to bind, allowing the vapor to penetrate more uniformly into the tissues (Guenther, 1972). In this work, the flowering and fruiting period occurred almost 
throughout the year, except in summer, when the plants were in the vegetative phase, as previously described (Brandão et al., 2015). This probably contributed to an increase in EO content in winter compared to the oil content extracted in summer in the fresh leaves of the BV2 $(1.1 \%)$ and PE3 $(0.9 \%)$ populations. The high rate of EO biosynthesis during the reproductive period in these populations may be due to the activation of enzymes required for the biosynthesis of certain compounds. The higher level of EO accumulated during flowering is a net result of the anabolic and catabolic processes during the several stages of flower development (Dubey $\&$ Luthra, 2001).

This research showed that the EOs chemical composition varied among the studied populations, highlighting the chemical diversity found in the same species. The common compounds in all populations were $\alpha$-thujene, $\alpha$-pinene, sabinene, $\alpha$-humulene, $(E)$-caryophyllene, spathulenol, myrcene, allo-aromadendrene, $\beta$-sesquiphellandrene, and $\alpha$-zingiberene, but with quantitative variations among them. A previous study on native populations of $V$. curassavica collected in Sergipe, Brazil, identified 53 compounds, of which 18 were also found in this study, except for $\alpha$-thujene, myrcene, $\beta$-cubebene, $\gamma$-muurolene, $(E)$-nerolidol, and $\beta$-copaen- $4 \alpha$-ol (Nizio et al., 2015). On the other hand, these compounds were obtained in $V$. curassavica genotypes cultivated in São Paulo, Brazil (Marques et al., 2019). These findings allow us to suggest that the diversity of compounds in the EO of $V$. curassavica may be influenced by geographic location compared to other studies (Santos et al., 2006; Nizio et al., 2015; Marques et al., 2019), as well as, the ability of the terpene synthase enzymes to convert the acyclic prenyl diphosphates and squalene into a multitude of cyclic and acyclic forms (Degenhardt et al., 2009). This property is found in almost half of the known monoterpenes and sesquiterpenes and may be attributed to the fact that the various reactive carbocationic intermediates can be stabilized in more than one way (Degenhardt et al., 2009). In this respect, we also observe that the chemical compounds' greatest diversity belongs to the classes of hydrocarbon sesquiterpenes and oxygenated sesquiterpenes compared to monoterpenes. Sesquiterpenes synthesized in the cytosol from farnesyl diphosphate by sesquiterpene synthases are structurally more diverse than monoterpenes due to the increased number of different cyclizations possible with five additional carbon atoms (Bohlmann et al., 1998; Nagegowda, 2010).

$\mathrm{O} \alpha$-humulene, an economically valuable constituent in the EO of $V$. curassavica, is a monocyclic sesquiterpene produced by terpene synthase enzymes using farnesyl pyrophosphate (Bohlmann et al., 1998). Its synthesis is related to the formation of $\mathrm{CO}_{2}$ and acquisition of photosynthesis intermediates (Dehsheikh et al., 2019). Variations in the content of $\alpha$-humulene (0.3-6.8\%) found in this work may result from the influence of genetic load and environmental conditions. The $\alpha$-humulene content was higher in the PE3 population $(2.0-6.8 \%)$, regardless of season and leaf drying. A previous study found higher $\alpha$-humulene content $(31.6 \%)$ in $V$. curassavica under field conditions with water and nutrition supply to the plants (Queiroz et al., 2020). In contrast, in our study, $V$. curassavica populations were established in sandy leached, and nutrient-poor soils, and plants were also exposed to high salinity, solar radiation, constant winds, and high soil temperatures. We also observed that all populations in summer, after the leaf drying process, maintained high $\alpha$-humulene contents $(0.7$ to $6.8 \%)$. Also, we found that $\gamma$-muurolene, $\alpha$-cubebene, and $\beta$-copaen- $4 \alpha$-ol were detected only after the leaves were dried. Similarly, Amaral et al. (2017) found an increase in sesquiterpene molecules after leaf drying when studying tree species from the Atlantic Forest. These authors attributed that such changes in the EOs chemical composition are due to the higher stability of sesquiterpenes compared to monoterpenes and oxidation processes during drying.

We found intrapopulation variability of the chemical compounds in the EOs of $V$. curassavica. This variability plays an important role in understanding natural populations, as they outline conservation and genetic improvement strategies. Thus, we distributed the plants into groups regardless of leaf drying. As a result, HCA grouped the 16 populations into three groups in summer and eight in winter. PCA identified that (E)-caryophyllene, $\alpha$-humulene, and allo-aromadendrene were strongly associated with populations PE1, PE3, BS2, PI2, BS4, and NA1 in summer. These populations were strongly related to climatic conditions in summer, such as Tp and $\mathrm{Pp}$. The seasonal dynamics of the municipalities where the collections were made are quite similar, with hot and rainy summer (average values $\mathrm{Tp}$ of $24.5{ }^{\circ} \mathrm{C}$; Pp of $240.1 \mathrm{~mm}$ ) and mild and dry winter (average values $\mathrm{Tp}$ of $17.3{ }^{\circ} \mathrm{C}$, Pp of $95.3 \mathrm{~mm}$ ). However, with increased sunlight in summer, photosynthesis tends to increase, and consequently, high levels of energy are available for plant growth and development (Rezaei et al., 2019), resulting in the balance of available energy being directed toward secondary metabolite production. Similarly, the higher $\mathrm{Tp}$ recorded in summer provides considerable effects on substrate concentrations because of its effect on modifying day length (Burbott \& Loomis, 1967).

The water availability is known to increase the production of terpenes (Maatallah et al., 2016) due to biosynthetic reactions occurring in an aqueous medium. Similar results were reported by Boira and Blanquer 
(1998), who revealed a positive relationship of sesquiterpenes, such as $\beta$-caryophyllene and caryophyllene oxide, when Tp and Pp increased. On the other hand, we observed that $\mathrm{Tp}$ and $\mathrm{Pp}$ in winter are related to sabinene and $\alpha$-thujene, although their relationships are less evident. These monoterpenes are inverse to $(E)$-caryophyllene, $\alpha$-humulene, and allo-aromadendrene in winter. This negative relationship between monoterpenes and sesquiterpenes can be interpreted as competition between two pathways for the same precursor (Ghaffari et al., 2018; Ghaffari et al., 2019). Thus, we can suggest that isopentenyl pyrophosphate fluxes are dominant to the plastid (site of monoterpene synthesis) under winter conditions, whereas this flux tends toward the cytosol (site of sesquiterpene synthesis) in summer (McCaskill \& Croteau, 1994).

Another factor considered in our work was the soil in which the plants grow. This is one of the main aspects that differentiated our research, contrasting with $V$. curassavica plants from different geographical origins and propagated under ideal growing conditions (Santos et al., 2006; Nizio et al., 2015; Marques et al., 2019). In this study, most of the collection sites belong to the order of arid soils. This order is typically defined by saline or alkaline soils with very little OM, characteristic of arid regions (Dewan \& Famouri, 1964). Most of the collection sites belong to the order of arid soils. This order is typically defined by saline or alkaline soils with very little OM, characteristic of arid regions (Dewan \& Famouri, 1964). As the habitats have distinct pedological characteristics, we identified that $\mathrm{pH}, \mathrm{OM}, \mathrm{S}$, and $\mathrm{V}$ are associated with the synthesis of $(E)$-caryophyllene, $\alpha$-humulene, and allo-aromadendrene from populations PE3, BV3, BS4, PI2, and BS2, acting inversely with Al in the soil. The positive effects of these chemical compounds associated with $\mathrm{pH}$ affect plant nutrient availability and natural soil fertility (OM, S, and V). On the other hand, by acting inversely with $\mathrm{Al}$, they decrease the toxic effects of this element on plants. Al impairs the synthesis of energy in the plant due to the inhibition of $\mathrm{P}$ uptake and transport and the ATPase enzyme activity (Ahn et al., 2001; Abichequer et al., 2003). Considering the need for energy in the form of ATP and other P-dependent enzymes in the synthesis of EOs (Loomis \& Corteau, 1972), the limitation of this element may be determinant in the increase of $(E)$-caryophyllene, $\alpha$-humulene and allo-aromadendrene contents under conditions of $\mathrm{Al}$ saturation in the soil. Thus, the chemical variation of EOs observed in $V$. curassavica populations is due to environmental conditions, as well as their interactions.

\section{Conclusion}

The PI1 and PE1 populations exhibit the highest EO content, while PE3 has the highest $\alpha$-hulene content regardless of drought and season. HCA demonstrates differences in the chemical profile of populations from different locations. PCA corroborates these findings and shows that $(E)$-caryophyllene, $\alpha$-humulene and allo-aromadendreno are related to soil and climate conditions (Tp, Pp, OM, SB, V and S) of PE1, PE3, BS2, Populations PI2, BS4 and NA1 in summer.

\section{References}

Abichequer, A. D., Bohnen, H., \& Anghinoni, I. (2003). Absorção, translocação e utilização de fósforo por variedades de trigo submetidas à toxidez de alumínio. Revista Brasileira de Ciência do Solo, 27, 373-378. https://doi.org/10.1590/S0100-06832003000200017

Adams, R. P. (2007). Identification of Essential Oil Components by Gas Chromatography/Quadrupole Mass Spectroscopy (p. 469). Allured, Illinois.

Ahn, S. J., Sivaguru, M., Osawa, H., Chung, G. C., \& Matsumoto, H. (2001). Aluminum inhibits the H+-ATPase activity by permanently altering the plasma membrane surface potentials in squash roots. Plant Physiology, 126, 1381-1390. https://doi.org/10.1104/pp.126.4.1381

Amaral, W. D., Deschamps, C., Bizzo, H. R., Pinto, M. A. S, Biasi, L. A., \& Silva, L. E. (2017). Essential oil yield and composition of native tree species from Atlantic Forest, South of Brazil. Journal of Essential Oil Bearing Plants, 20, 1525-1535. https://doi.org/10.1080/0972060X.2017.1346484

Bayeux, M. C., Fernandes, A. T., Foglio, M. A., \& Carvalho, J. E. (2002). Evaluation of the antiedematogenic activity of artemetin isolated from Cordia currassavica D.C. Brazilian Journal of Medical and Biological Research, 35, 1229-1232. https://doi.org/10.1590/S0100-879X2002001000017

Bohlmann, J., Meyer-Gauen, G., \& Croteau, R. (1998). Plant terpenoid synthases: molecular biology and phylogenetic analysis. Proceedings of the National Academy of Sciences, 95, 4126-4133. https://doi.org/ 10.1073/pnas.95.8.4126

Boira, H., \& Blanquer, A. (1998). Environmental factors affecting chemical variability of essential oils in Thymus piperella L. Biochemical Systematics and Ecology, 26, 811-822. https://doi.org/10.1016/ S0305-1978(98)00047-7 
Bouyahya, A., Belmehdi, O., El Jemli, M., Marmouzi, I., Bourais, I., Abrini, J., .. Bakri, Y. (2019). Chemical variability of Centaurium erythraea essential oils at three developmental stages and investigation of their in vitro antioxidant, antidiabetic, dermatoprotective and antibacterial activities. Industrial Crop and Products, 132, 111-117. https://doi.org/10.1016/j.indcrop.2019.01.042

Brandão, D. S., Mendes, A. D. R., Santos, R. R., Rocha, S. M. G., Leite, G. L. D., \& Martins, E. R. (2015). Biologia floral e sistema reprodutivo da erva-baleeira (Varronia curassavica Jacq.). Revista Brasileira de Plantas Medicinais, 17, 562-569. https://doi.org/10.1590/1983-084X/14_011

Burbott, A. J., \& Loomis, W. D. (1967). Effects of light and temperature on the monoterpenes of peppermint. Plant Physiology, 42, 20-28. https://doi.org/10.1104/pp.42.1.20

Carvalho, J. R., Rodrigues, R. F. O., Sawaya, A. C. H. F., Marques, M. O. M., \& Shimizu, M. T. (2004). Chemical composition and antimicrobial activity of the essential oil of Cordia verbenacea D.C. Journal of Ethnopharmacology, 95, 297-301. https://doi.org/10.1016/j.jep.2004.07.028

De Almeida, L. F. R., De Oliveira, P. R, Bufalo, J., Marques, M. O. M., Facanali, R., \& Frei, F. (2016). Non-oxygenated sesquiterpenes in the essential oil of Copaifera langsdorffii Desf. Increase during the day in the dry season. PLoS One, 11, e0149332. https://doi.org/10.1371/journal.pone.0149332

Degenhardt, J., Kollner, T. G., \& Gershenzon, J. (2009). Monoterpene and sesquiterpene synthases and the origin of terpene skeletal diversity in plants. Phytochemistry, 70, 1621-1637. https://doi.org/10.1016/j.phytochem. 2009.07.030

Dehsheikh, B. A., Sourestani, M. M., Dehsheikh, P. B., Vitalini, S., Iriti, M., \& Mottaghipisheh, J. (2019). A comparative study of essential oil constituents and phenolic compounds of Arabian Lilac (Vitex trifolia var. purpurea): An evidence of season effects. Foods, 8, 52-57. https://doi.org/10.3390/foods8020052

Dubey, V. S., \& Luthra, R. (2001). Biotransformation of geranyl acetate to geraniol during palmarosa (Cymbopogon martinii Roxb: Wats var. motia) inflorescence development. Phytochemistry, 57, 675-680. https://doi.org/10.1016/s0031-9422(01)00122-4

Feijó, E. V., Da, S., Oliveira, R. A. D., Costa, L. C., \& Do, B. (2014). Light affects Varronia curassavica essential oil yield by increasing trichomes frequency. Revista Brasileira Farmacognosia, 24, $516-523$. https://doi.org/10.1016/j.bjp.2014.10.005

Fernandes, E. S., Passos, G. F., Medeiros, R., Da Cunha, F. M., Ferreira, J., Campos, M. M., Pianowski, L. F., \& Calixto, J. B. (2007). Anti-inflammatory effects of compounds alpha humulene and (-)-trans-caryophyllene isolated from the essential oil of Cordia verbenacea. European Journal of Pharmacology, 569, 228-236. https://doi.org/10.1016/j.ejphar.2007.04.059

Ghaffari, Z., Rahimmalek, M., \& Sabzalian, M. R. (2018). Variations in essential oil composition and antioxidant activity in Perovskia abrotanoides Kar. Collected from different regions in Iran. Chemical Biodiversity, 15, e1700565. https://doi.org/10.1002/cbdv.201700565

Ghaffari, Z., Rahimmalek, M., \& Sabzalian, M. R. (2019). Variation in the primary and secondary metabolites derived from the isoprenoid pathway in the Perovskia species in response to different wavelengths generated by light emitting diodes (LEDs). Industrial Crop and Products, 140, 111592. https://doi.org/ 10.1016/j.indcrop.2019.111592

Guenther, E. (1972). The essential oils (pp. 453-454). Krieger Publishing Company, New York.

Hernández, T., Hernández, D., Orozco, J., Serrano, R., Duran, A., Meraz, S., ... Avila, G. (2014). Temporal variación de la composición química y la actividad antimicrobiana del aceite esencial de Cordia curassavica (Jacq.) Roemer y Schultes: Boraginaceae. Boletín Latinoamericano y del Caribe de Plantas Medicinales y Aromáticas, 13, 100-108.

Hoeltgebaum, M. P., Montagna, T., Lando, A. P., Puttkammer, C., Orth, A. I., Guerra, M. P., \& Reis, M. S. D. (2018). Reproductive biology of Varronia curassavica Jacq. (Boraginaceae). Anais da Acadêmia Brasileira de Ciencias, 90, 59-71. https://doi.org/10.1590/0001-3765201820160273

Loomis, W. D., \& Corteau, R. (1972). Essential oil biosynthesis. Recent Advances in Phytochemistry, 6, $147-185$. https://doi.org/10.1016/B978-0-12-612406-4.50012-7

Maatallah, S., Nasri, N., Hajlaoui, H., Albuch, A., \& Elaissi, A. (2016). Evaluation changing of essential oil of laurel (Laurus nobilis L.) under water deficit stress conditions. Industrial Crop and Products, 91, 170-178. https://doi.org/10.1016/j.indcrop.2016.07.001 
Magalhães, P. M. (2010). Estratégias para o mercado de plantas medicinais e aromáticas no Brasil: O exemplo da erva-baleeira. Informe Agropecuário, 31, 94-100. https://doi.org/10.1016/j.indcrop.2016.07.001

Marques, A. P., Bonfim, F. P. G, Dantas, W. F. C., Puppi, R. J., \& Marques, M. O. M. (2019). Chemical composition of essential oil from Varronia curassavica Jacq. accessions in different seasons of the year. Industrial Crop and Products, 140, 111656. https://doi.org/10.1016/j.indcrop.2019.111656

Mccaskill, D., \& Croteau, R. (1994). Recent advances in terpenoid biosynthesis: Implications for essential oil production. In N. Verlet (Ed.), 4 emes rencontres internationals (pp. 92-102). Nyons, France.

Mclafferty, F. W., \& Stauffer, D. B. (1994). Wiley Registry of Mass Spectral Data (6th ed., p. 508). Wiley Interscience, New York, NY.

Nagegowda, D. A. (2010). Plant volatile terpenoid metabolism: Biosynthetic genes, transcriptional regulation and subcellular compartmentation. FEBS Lett, 584, 2965-2973. https://doi.org/10.1016/j.febslet.2010. 05.045

NIST (National Institute of Standards and Technology). (2018). NIST Standard Reference Database Number 69. Linstrom P. J., \& Mallard, W. G. (Eds.), NIST Chemistry Webbook. NIST-USDC. https://doi.org/ 10.18434/T4D303

Nizio, D. A. C., Brito, F. A., Sampaio, T. S., Melo, J. O., Silva, F. L. S., Gagliardi, P. R., ... Blank, A. F. (2015). Chemical diversity of native populations of Varronia curassavica Jacq: and antifungal activity against Lasiodiplodia theobromae. Industrial Crop and Products, 76, 437-448. https://doi.org/10.1016/j.indcrop. 2015.07 .026

Oliveira, M. (2017). Laboratório em renovação. Revista Pesquisa Fapesp, 255, 74-77.

Oliveira, U., Soares-Filho, B. S., Santos, A. J., Paglia, A. P., Brescovit, A. D., Carvalho, C. J. B., ... Ferro, V. G. (2019). Modelling highly biodiverse areas in Brazil. Nature Scientific Reports, 9, 6355. https://doi.org/ 10.1038/s41598-019-42881-9

Passos, G. F., Fernandes, E. S., Da Cunha, F. M., Ferreira, J., Pianowski, L. F., Campos, M. M., \& Calixto, J. B. (2007). Anti-inflammatory and anti-allergic properties of the essential oil and active compounds from Cordia verbenacea. Journal of Ethnopharmacology, 110, 323-333. https://doi.org/10.1016/j.jep.2006.09.032

Queiroz, T. B., Fonseca, F. S. A., Mendes, A. D. R., Azevedo, A. M., \& Martins, E. R. (2019). Chemical diversity of accessions of the in vivo germplasm bank of Varronia curassavica (Jacq.). Acta Scientiarum Agronomy, 42, e42726. https://doi.org/10.4025/actasciagron.v42i1.42726

R DCT. (2012). R: A language and environment for statistical computing. Vienna: R Foundation for Statistical Computing. Retrieved from https://http://www.r-project.org

Rahimmalek, M., \& Goli, S. A. H. (2013). Evaluation of six drying treatments with respect to essential oil yield, composition and color characteristics of Thymys daenensis subsp. Daenensis Celak leaves. Industrial Crop and Products, 42, 613-619. https://doi.org/10.1016/j.indcrop.2012.06.012

Rahimmalek, M., Heidari, E. F., Ehtemam, M. H., \& Mohammadi, S. (2017). Essential oil variation in Iranian Ajowan (Trachyspermum ammi (L.) Sprague) populations collected from different geographical regions in relation to climatic factors. Industrial Crop and Products, 95, 591-598. https://doi.org/10.1016/j.indcrop. 2016.11.017

Rezaei, M., Razmjoo, J., Ehtemam, M. H., Karimmojeni, H., \& Zahedi, M. (2019). The interaction between shade and drought affects essential oil quantity and quality of Vitex agnus-castus L. leaves and seeds. Industrial Crop and Products, 137, 460-467. https://doi.org/10.1016/j.indcrop.2019.05.059

Ribeiro, M. C., Martensen, A. C., Metzger, J. P., Tabarelli, M., Scarano, F., \& Fortin, M. (2011). Chapter 21. The Brazilian Atlantic Forest: a shrinking biodiversity hotspot. In F. E. Zachos, \& J. C. Habel (Eds.), Biodiversity Hotspots. Springer-Verlag, Berlin Heidelberg. https://doi.org/10.1007/978-3-642-20992-5_21

Roldão, E. F., Witaicenis, A., Seito, L. N., Hiruma-Lima, C. A., \& Di Stasi, L. C. (2008). Evaluation of the antiulcerogenic and analgesic activities of Cordia verbenacea DC. (Boraginaceae). Journal of Ethnopharmacology, 119, 94-98. https://doi.org/10.1016/j.jep.2008.06.001

Santos, R. P., Nunes, E. P., Nascimento, R. F., Santiago, G. M. P., Menezes, G. H. A., Silveira, E. R., \& Pessoa, O. D. L. (2006). Chemical composition and larvicidal activity of the essential oils of Cordia leucomalloides and Cordia curassavica from the Northeast of Brazil. Journal of the Brazilian Chemical Society, 17, 1027-1030. https://doi.org/10.1590/S0103-50532006000500030 
Silva, S. C. (1999). Manual de Análises Químicas de Solos, Plantas e Fertilizantes (1st ed., p. 624). Embrapa: Brasília.

Souza, M. T., Souza, M. T., Bernardi, D., Krinski, D., Melo, D. J., Oliveira, D. C., ... Zawadneak, M. A. C. (2020). Chemical composition of essential oils of selected species of Piper and their insecticidal activity against Drosophila suzukii and Trichopria anastrephae. Environmental Science and Pollution Research, 27, 13056-13065. https://doi.org/10.1007/s11356-020-07871-9

Souza, M. T., Souza, M. T., Bernardi, D., Melo, D. J., Zarbin, P. H. G., \& Zawadneak, M. A. C. (2021). Insecticidal and oviposition deterrent effects of essential oils of Baccharis spp. and histological assessment against Drosophila suzukii (Diptera: Drosophilidae). Nature Scientific Reports, 11, 3944. https://doi.org/ 10.1038/s41598-021-83557-7

Van Den Dool, H., \& Kratz, P. D. (1963). A generalization of the retention index system including linear temperature programmed gas-liquid partition chromatography. Journal Chromatography A, 11, 463-471. https://doi.org/10.1016/S0021-9673(01)80947-X

\section{Copyrights}

Copyright for this article is retained by the author(s), with first publication rights granted to the journal.

This is an open-access article distributed under the terms and conditions of the Creative Commons Attribution license (http://creativecommons.org/licenses/by/4.0/). 